\title{
Intramolecular dynamics. II. Artificial intelligence search evaluation function and treatment of resonance centers for large systems
}

\author{
Yongfeng Zhang and R. A. Marcus \\ Arthur Amos Noyes Laboratory of Chemical Physics, California Institute of Technology, Pasadena, \\ California $91125^{\text {) }}$
}

(Received 20 December 1990; accepted 6 January 1992)

\begin{abstract}
The role of resonance centers in obtaining large size matrices via an artificial intelligence (AI) search is discussed. The results are used to develop for an AI search an evaluation function which takes cognizance of isolated internal resonances in these many-state systems. The effect of later-accepted states on resonance centers is included. This evaluation function and one without a resonance factor are used in Part III of this series. A search is made there to select zeroth-order vibrational states of benzene that are used in a diagonalization treatment of $\mathrm{CH}$ overtone spectra. When there are many overlapping resonances, as in that case, a comparison of results obtained with those two types of evaluation function is of particular interest.
\end{abstract}

\section{INTRODUCTION}

It is well known that the properties, rate, and mechanism of intramolecular vibrational relaxation (IVR) play a fundamental role in many areas of chemical dynamics. ${ }^{1,2}$ Insight into aspects of Rice-Ramsperger-Kassel-Marcus (RRKM) and other treatments of unimolecular reactions, ${ }^{2-4}$ multiphoton processes, ${ }^{5}$ mode selective processes, ${ }^{6}$ and overtone-induced reactions ${ }^{1}$ all involve elucidation of the IVR behavior.

A basis-set quantum-mechanical study of vibrational spectra and intramolecular dynamics in large or moderately large molecules is often limited by the computational memory and time associated with the use of many zeroth-order states in high-dimensional systems. For example, the $\mathbf{C}-\mathbf{H}$ overtone $v=3$ spectrum for benzene ${ }^{7}$ involves potentially millions of possible zeroth-order states, the density of vibrational states in the corresponding energy range being around 420 states per $\mathrm{cm}^{-1}$ for the in-plane vibrations alone. Including them constitutes one of the main difficulties in studies of intramolecular dynamics. However, the use of artificial intelligence (AI) search methods ${ }^{8-12}$ offers one possible procedure for limiting the number of states to the more relevant ones, and we have used it in our recent work. ${ }^{8-10}$ Several papers have appeared in which AI techniques have been used to solve various problems by Wyatt, Chu, and others, for example, in multiphoton dynamics, ${ }^{11}$ vibrational eigenvalues, ${ }^{13}$ computational physics, ${ }^{14}$ and organic syntheses. ${ }^{15}$ These papers have shown the feasibility of a variety of AI methods in their respective applications. Once the subset of states is found the characteristics of vibrational spectrum and dynamics can be treated using this reduced set of states to diagonalize the Hamiltonian. Other efficient computational techniques for treating molecules with many states are also being employed. ${ }^{16}$

In our current series of studies there is such a large number of states that internal resonances are common. Accordingly, in the present work we consider a resonance effect on

\footnotetext{
") Contribution No. 8370 .
}

the procedure of the $\mathrm{AI}$ search in which the role of resonance centers for a given coupling tier and for the shift relations for these centers is discussed.

When there are many overlapping resonances, as in Part III of this series, it is not certain, a priori, that an evaluation function based on isolated resonances is an improvement over one which is not. Results obtained with resonant and nonresonant types of evaluation functions are given in Part III.

\section{SEARCH TECHNIQUE}

In an AI search method, a search algorithm and an evaluation function are employed. The relevant states of the system in the zeroth-order approximation may be termed the initial states or tier-1 states. In intramolecular dynamics problems the occupation probability in the initial states will become distributed in time over many final zeroth-order states. We define a tier-2 state as a state that couples to a tier1 state and whose evaluation function is above a minimum value. A tier- $n$ state can be similarly defined via its coupling to a tier- $(n-1)$ state. Thus, a tier- $n$ state either does not couple to a tier- $(n-2)$ or to a lower tier state, or the couplings are so weak that the corresponding evaluation function is less than the assigned minimum. In this way, the the zeroth-order states form paths, a tier- $(n-1)$ state being the parent state of a ticr- $n$ state. An intuitive picture is given in Ref. 8 or in the present Fig. 1.

The influence of a single state on the dynamics along the path can be quite complicated. Sometimes, if any state in a relevant path is deliberately excluded, the description of the dynamics can be dramatically changed, for example, when the paths to a significant state are so broken. In an AI search the more important continuous paths are selected to form the subset of important states. The Hamiltonian is then diagonalized with this subset of statcs. ${ }^{8,9}$

In AI terminology, the Hamiltonian can be used to form a special operator, the successor operator, which when applied to a chosen state, and appropriate matrix elements are calculated, yields all states that can be directly reached in a single step from the chosen state. The search algorithm de- 
termines the order in which possible zeroth-order states (and paths) are considered, and an evaluation function is used to provide an estimate of the importance of the possible zeroth-order states. For intramolecular dynamics problems, beam and best-first searches ${ }^{8-12}$ are suitable. In a beam search all possible paths are considered from every newly found state whose evaluation function is above a minimum value. A best-first search considers the most promising of all incomplete paths first. As in the earlier study it is practical to use a compromise ${ }^{8-10}$ whereby a beam search is performed for the first two tiers of searching, so as to obtain a breadth for the initial part of the search, and a best-first search is utilized thereafter.

We consider a path shown in Fig. 1. In previous work $^{8-10}$ the proposed evaluation function was motivated by perturbation theory. The value $C_{n}$ of a tier- $n$ state in a path for one of the evaluation functions used there is given by

$$
C_{n}=\prod_{i=2}^{n} \frac{\left|a_{i-1, i}\right|}{\Delta E_{i 1}+\Delta E_{i, i-1}},
$$

where

$$
\begin{aligned}
& \Delta E_{i 1}=\left|a_{i i}-a_{11}\right| \\
& \Delta E_{i, i-1}=\left|a_{i i}-a_{i-1, i-1}\right|,
\end{aligned}
$$

and the $a_{i i}$ denote the energies of the basis set states, while $a_{i-1, i}$ denotes the coupling element of the $i$ th state to its parent state, as shown in Fig. 1.

For the systems with a low density of states, the internal resonance among states is not very important, and so Eq. (1) was satisfactory, as in Refs. 8 and 9. However, for systcms such as benzene at moderate to high energies, the density of states is so high that internal resonance among states is a frequent occurrence. In this case, very small couplings may play an important role. The evaluation function described below is based on the behavior of isolated resonance centers.

\section{EVALUATION FUNCTION MODIFIED BY INTERNAL RESONANCES}

Motivated by the analysis given later in Secs. IV and V, an evaluation function, a dimensionless quantity, which is a

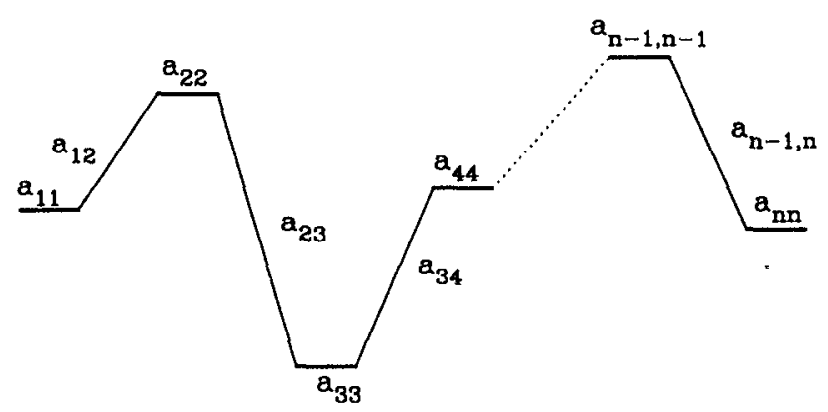

Tier 1 Tier 2 Tier 3 Tier 4 Tier $n-1$ Tier $\mathbf{n}$

FIG. 1. A single path involving $n$ states. generalization of Eq. (1), is given by

$C_{n}=W_{2}\left[\prod_{i=2}^{n}\left|a_{i-1, i}\right| f \xi\left(a_{i-1, i-1}, a_{i i}\right)\right] \eta\left(a_{33}\right)$,

where $W_{2}$ is the weight factor of the tier-2 state in the path, defined later in Eq. (17); $f$ is the tier factor, which describes a decay effect along the path chain; $\xi$ has a form such as that in Eq. (1), or one for parallel circuits, or other variants: ${ }^{9}$

$$
\begin{aligned}
\xi\left(a_{i-1, i-1}, a_{i i}\right) & =\frac{1}{\left(\Delta E_{i 1}+\Delta E_{i, i-1}\right)}, \\
\xi\left(a_{i-1, i-1}, a_{i i}\right) & =\frac{1}{2}\left[\frac{1}{\Delta E_{i 1}}+\frac{1}{\Delta E_{i, i-1}}\right],
\end{aligned}
$$

where $\Delta E_{i j}$ is the absolute value of the energy difference between states $i$ and $j,\left|E_{i}-E_{j}\right|$. Finally, $\eta$ is a dimensionless factor which characterizes the proximity of a tier- 3 state to being in resonance with a tier- 1 state. Two alternative examples of functional forms for $\eta$ are

$$
\eta\left(a_{33}\right)= \begin{cases}\alpha & \text { if }\left|a_{33}-R_{3}\right|<\tau \\ \beta & \text { if } \tau \leqslant\left|a_{33}-R_{3}\right| \leqslant 5 \tau \\ 1.0 & \text { if }\left|a_{33}-R_{3}\right|>5 \tau\end{cases}
$$

and

$$
\eta\left(a_{33}\right)=1+A \exp \left[-B\left|a_{33}-R_{3}\right| / \tau\right],
$$

where $\alpha>\beta>1, a_{33}$ is the energy of the zeroth-order tier-3 state, $R_{3}$ denotes the value that $a_{33}$ would have for exact resonance, i.e., $R_{3}$ is the resonance center for tier-3 states, which is given later in Eqs. (11) and (13); $\tau$ is the width of the resonance (width at half-height) given later by Eqs. (12) and (14). In applying Eqs. (3) and (5) in Part III, we chose some preassigned values for $\alpha, \beta$, and $f$ or $A, B$ and $f$.

In the application of Eq. (3) to Part III, we included only the influence of the resonances between tier- 1 and tier- 3 states, coupled via off-resonant tier- 2 states. Higher order resonant couplings exist, e.g., tier-1 and tier-4 states coupled via off-resonant tier- 2 and tier- 3 states, but they were omitted in our initial efforts to consider the effect of resonances on the AI search. Equation (1) follows from Eq. (3) when one sets $W_{2}=f=\eta=1$ and uses Eq. (4a) for $\xi$.

\section{RESONANCE CENTER AND ITS SHIFT AND WIDTH}

\section{A. Resonance center}

To motivate the evaluation function given by Eqs. (3) to (5), we consider the effect of a resonance center on the coupling between a tier- 1 and a tier- 3 state. In multistate systems the effect of a particular zeroth-order state on the final eigenstates depends both on the couplings between this state and other states and on the energy levels of these zerothorder states. The 3-state system provides a useful illustration for the role of the resonance in a 3-tier search.

We consider a 3 -state system $\left\{\phi_{1}, \phi_{2}, \phi_{3}\right\}$, where $\phi_{1}$ corresponds the initial state (i.e., tier- 1 state) and $\phi_{2}$ and $\phi_{3}$ denote a tier- 2 state and a tier- 3 state, respectively. The eigenstates for this system $\psi_{i}$ can be written as $\sum_{j=1}^{3} c_{i j} \phi_{j}$, where $i=1,2,3 . \psi_{i}$ denotes the eigenstate in which the component $\phi_{i}$ is dominant. We write the Hamiltonian for this 3state system as 


$$
\mathbf{H}=\left(\begin{array}{ccc}
a_{11} & a_{12} & 0 \\
a_{12} & a_{22} & a_{23} \\
0 & a_{23} & a_{33}
\end{array}\right),
$$

i.e., these tier- 1 and tier-3 states are coupled only via a tier-2 state.

For illustrative purposes, the values $a_{11}=8800 \mathrm{~cm}^{-1}$, $a_{22}=9000 \mathrm{~cm}^{-1}$, and $a_{12}=20 \mathrm{~cm}^{-1}$ are used, values which are typical for the $v=3$ overtone of a $\mathrm{CH}$ stretching mode in benzene. We next examine, through the quantity $c_{11}^{2}$, the dependence of the eigenvector $\psi_{1}$ of the matrix $\mathbf{H}$ and of the corresponding eigenvalue $\epsilon_{1}$, upon $a_{33}$, for several values of $a_{23}$. ( $c_{11}^{2}$ reflects the $\phi_{1}$ contents in the $\phi_{1}$-rich state $\psi_{1}$.) The results show that in a very narrow range of values of $a_{33}$, the coefficient $c_{11}$, and the eigenvalue of the $\phi_{1}$-dominant state change extremely rapidly with $a_{33}$. The more $c_{11}^{2}$ decreases from unity, the more $\phi_{1}$ can contribute to $\psi_{3}$ and so be propagated along a path involving state 3 . The range in which $c_{11}^{2}$ changes very rapidly with $a_{33}$ is termed the resonance range, and the value of $a_{33}$ for which $c_{11}^{2}$ is a minimum (typically cusplike as in Figs. 2 and 3 ) will be termed the resonance center and denoted by $R_{3}$. If $a_{33}$ is located in the resonance range a very small value of the product of the $\left(\phi_{1}, \phi_{2}\right)$ and $\left(\phi_{2}, \phi_{3}\right)$ couplings, $a_{12} a_{23}$, will strongly influence the population $c_{11}^{2}$ of $\phi_{1}$ in $\psi_{1}$ (by definition of a resonance), and propagate the influence of $\phi_{1}$ along the path involving state 3 . For example, if $a_{33}$ occurs at the resonance center $R_{3}$, in the examples cited below, where $a_{12}=20$ $\mathrm{cm}^{-1}$, an $a_{23}$ which is even as small as $0.5 \mathrm{~cm}^{-1}$ can reduce population of $\phi_{1}$ in $\psi_{1}$ by as much as $40 \%$, and when as in the case studied $\phi_{2}$ is off resonant, it will correspondingly increase the population of $\phi_{1}$ in $\psi_{3}$.

If $a_{23}$, the coupling between a tier-2 state and tier-3 state, is small the center of the resonance range $R_{3}$, defined above, is almost independent of $a_{23}$, as seen in Fig. 2. For this simple case that $a_{23}$ is small, the value of $a_{33}$ for which $c_{11}^{2}$ will be cusplike, namely $R_{3}$, is simply equal to the energy of

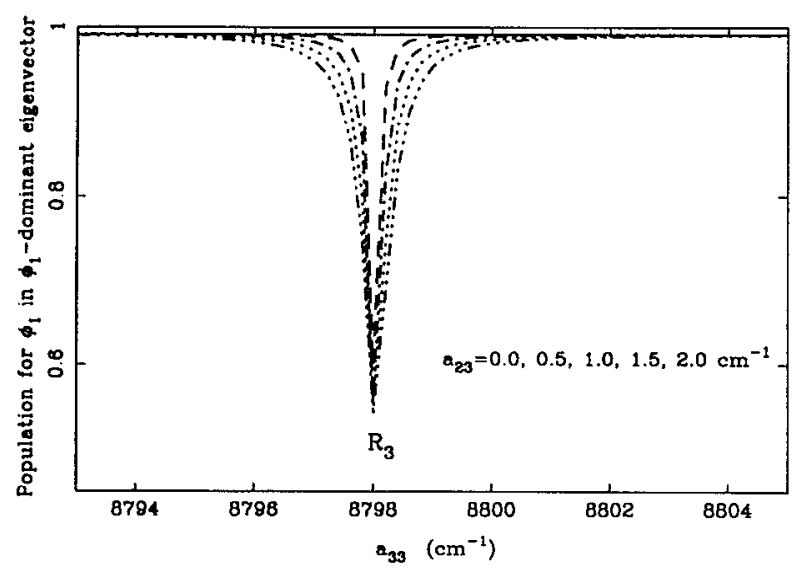

FIG. 2. Resonance centers of 3-state systems for weak coupling, the notation is defined in Eq. (3), $a_{11}=8800, a_{22}=9000, a_{12}=20 \mathrm{~cm}^{-1}$.

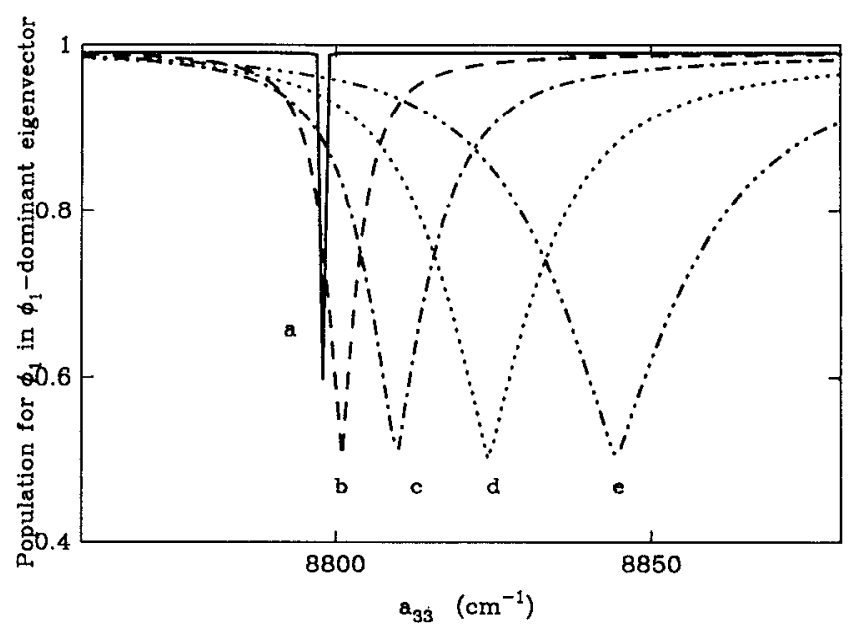

FIG. 3. Population analysis $c_{11}^{2}$ of the $\phi$-dominant eigenfunction of 3-state systems for strong couplings. The notation is defined in Eq. (3), $a_{11}=8800, a_{22}=9000, a_{12}=20 \mathrm{~cm}^{-1} \cdot a_{23}=0.5,24.5,48.5,72.5$, and $96.5 \mathrm{~cm}^{-1}$, respectively for (a)-(e). The resonance center occurs for these cases, with a resonance center shift of $0.0,3.0,11.8,25.9$, and $46.5 \mathrm{~cm}^{-1}$, respectively. The half width at half height of resonance center range for the last four strong couplings are $\tau=6.0,11.9,18.0$, and $24.8 \mathrm{~cm}^{-1}$, respectively. The population is the square of the coefficient $c_{11}$ of $\phi_{1}$ in the $\phi_{1}$-dominant eigenstate $\psi_{1}$.

the $\phi_{1}$-dominant state in the two-state $\left(\phi_{1}, \phi_{2}\right)$ system, denoted by $\alpha_{1}$,

$$
R_{3} \cong \alpha_{1},
$$

where $\alpha_{1}$ is obtained from the standard two-state perturbation expression

$$
\begin{gathered}
\alpha_{1}=\frac{1}{2}\left\{\left(a_{11}+a_{22}\right)+\left(a_{11}-a_{22}\right)\right. \\
\left.\times\left[1+\frac{4 a_{12}^{2}}{\left(a_{11}-a_{22}\right)^{2}}\right]^{1 / 2}\right\} . \\
\text { If }\left|a_{12} / a_{22}-a_{11}\right| \text { is small, we have } \\
\alpha_{1} \approx a_{11}-\frac{a_{12}^{2}}{a_{22}-a_{11}} .
\end{gathered}
$$

For the actual system, $\alpha_{1}$ was obtained by the generalization of Eq. ( 8 ), namely by a diagonalization of the tier-1 plus tier2 states.

\section{B. Shift and width of resonance centers in a simple path}

A path is called simple if along the path each parent state couples with only one state in the next tier. Figure 1 provides an example of simple paths. If the coupling $a_{23}$ between the state 2 and 3 in a simple path is relatively large, the resonance center $R_{3}$ will be dependent on $a_{23}$, so that there is a shift of the resonance center relative to the case where $a_{23} \rightarrow 0$, as seen in the curves a to e in Fig. 3, where the curves a-e correspond to $a_{23}=0.5,24.5,48.5,72.5$, and 96.5 $\mathrm{cm}^{-1}$, respectively. The resonance center for the curve a represents $\alpha_{1}$, since $a_{23}$ is so small. The shift $R_{3}-\alpha_{1}$ is seen to be larger the larger $a_{23}$. 
We first consider the simple path, and then consider the case where a tier-1 state is coupled to more than one tier-3 state ("tree path"). To make a rough estimate of $R_{3}-\alpha_{1}$ for a simple path, we make use, in Appendix A, of a partitioning method, which is particularly suited to a description of a resonance where states are coupled via an off-resonant state or states. In Eq. (A4) of Appendix A, it is noted that exact resonance occurs when $\alpha_{1}$ equals to $\alpha_{3}, \alpha_{1}$ and $\alpha_{3}$ being the eigenvalues of the tier- 1 and tier- 3 states, as modified by the presence of the tier-2 state [cf. definition in Eq. (A3) of Appendix A]. The value of $a_{33}$ which satisfies $\alpha_{1}=\alpha_{3}$ is the exact position of the resonance center and is denoted by $R_{3}$. From Eq. (A4) there we obtain (setting $E \cong \alpha_{1}$ in the denominators in Eq. (A4),

$$
R_{3}-\alpha_{1} \cong \frac{a_{23}^{2}}{a_{22}-\alpha_{1}} .
$$

In a perturbation approximation one can set $\alpha_{1} \approx a_{11}$ in the denominator of Eq. (10) and the latter equation then reduces to

$$
R_{3}-\alpha_{1}=\frac{a_{23}^{2}}{a_{22}-a_{11}} .
$$

However, in practice, Eq. (10) is used in Part III rather than (11), since $\alpha_{1}$ is available from a prediagonalization.

Equations (10) and (11) are seen in Table I to provide a good approximation for calculating the shift of a resonance center caused by a large coupling $a_{23}$ between tier-2 states and tier-3 states. In Table I, a comparison is given between the computational results and those calculated from Eqs. (10) and (11) for the cases given in Fig. 4. The results in Table I demonstrate that both Eqs. (10) and (11) are in good agreement with these computational results.

The width of the resonance range depends upon the coupling elements $a_{23}$ and $a_{12}$, and on other factors. The partitioning method used in the Appendix A shows that the width of the resonance range at half-height, denoted by $\tau$, is given approximately by

$$
\tau \cong \frac{4}{\sqrt{3}}\left|\frac{a_{12} a_{23}}{a_{22}-a_{11}}\right| .
$$

TABLE I. Shift $R_{3}-R_{2}$ of resonance center for 3-state systems.

\begin{tabular}{cccc}
\hline \hline & \multicolumn{3}{c}{$R_{3}-R_{2}$, shift of resonance center } \\
\cline { 2 - 4 }$a_{23}$ & $\begin{array}{c}\text { Computational } \\
\text { results }\end{array}$ & $\begin{array}{c}\text { Calc. by } \\
\text { Eq. (10) }\end{array}$ & $\begin{array}{c}\text { Calc. by } \\
\text { Eq. (11) }\end{array}$ \\
\hline 24.5 & 2.92 & 2.97 & 3.00 \\
48.5 & 11.7 & 11.6 & 11.8 \\
72.5 & 26.1 & 26.0 & 26.3 \\
96.5 & 46.4 & 46.1 & 46.6 \\
\hline \hline
\end{tabular}

All numbers are in $\mathrm{cm}^{-1}$. The matrix is given by Eq. (1), with $a_{11}=8800$, $a_{22}=9000$, and $a_{12}=20 \mathrm{~cm}^{-1}$. For this case $R_{2}=8798 \mathrm{~cm}^{-1}$.

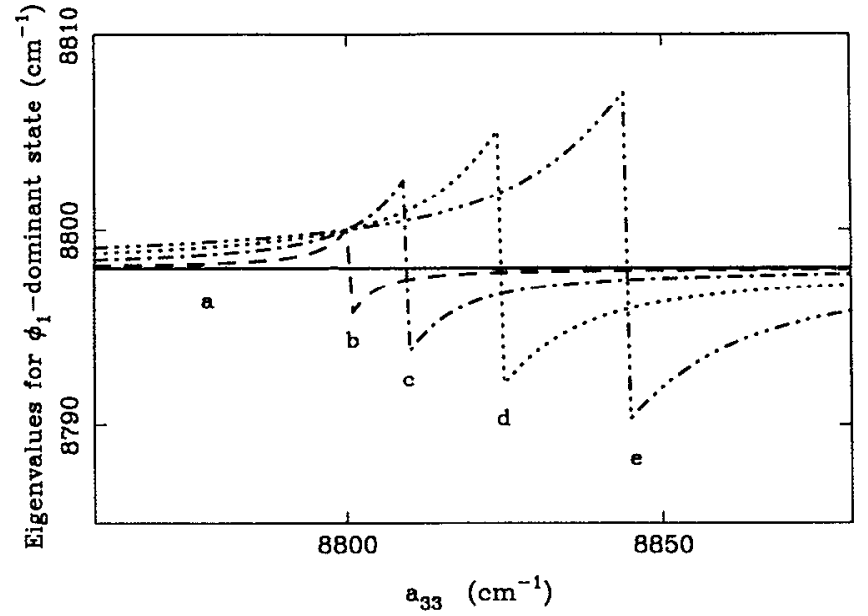

FIG. 4. Eigenvalues in resonance regions of 3-state systems for strong couplings. The notation is defined in Eq. (3), $a_{11}=8800, a_{22}=9000, a_{12}=20$ $\mathrm{cm}^{-1}$, and $a_{23}=0.5,24.5,48.5,72.5$, and $96.5 \mathrm{~cm}^{-1}$, respectively for (a)(e). Curve $a$ is the solid line.

For the examples given in this paper, $a_{12} /\left(a_{22}-a_{11}\right)$ is fixed, $a_{12}=20$ and $a_{22}-a_{11}=200$, so that

$$
\tau \cong \frac{1}{4.33}\left|a_{23}\right|,
$$

which is a good approximation to the results in Figs. 2 and 3.

It is expected, of course, that there will be substantial "jump" in the eigenvalues at the resonance centers, as illustrated in Fig. 4. The nature of the state changes there, the jump is, in effect, from one adiabatic state to another at the avoided crossing which occurs at the resonance center.

A path accepted in the AI search procedure may contain higher tier states. In our experience for a complicated system such as benzene, tier- 6 states or tier-7 states may have to be considered. Nevertheless, we have used a factor $\eta$ in Eq. (3) only for resonances coupling tier-1 and tier-3 states via off-resonant tier-2 states.

\section{Shift of resonance centers in a tree path}

In practical problems, a tier- 2 state may couple with several tier-3 states, as illustrated in Fig. 5. A path, in which several states may share one common parent state, was called a tree path. For completeness, we derive in Appendix $\mathrm{B}$ an expression for the resonance center $R_{3}$ and for the width for tree paths. However, in our present procedure used in Part III only the simple path formulas for $R_{3}$ and $\tau$ were used.

As shown in Appendix B, the partitioning method is applicable to this tree path case, there now being more than one tier-3 state contributing to the matrices $M_{a b}$ and $M_{b b}$ in Appendix A. If we take $\phi_{1}$-rich eigenvalue of $M_{a a}$ to still be given by $\alpha_{1}$, it is shown in Appendix B that a resonance center at the tier-3 stage for a tree path having $K$ tier-3 states is located, to a first-order approximation, at 


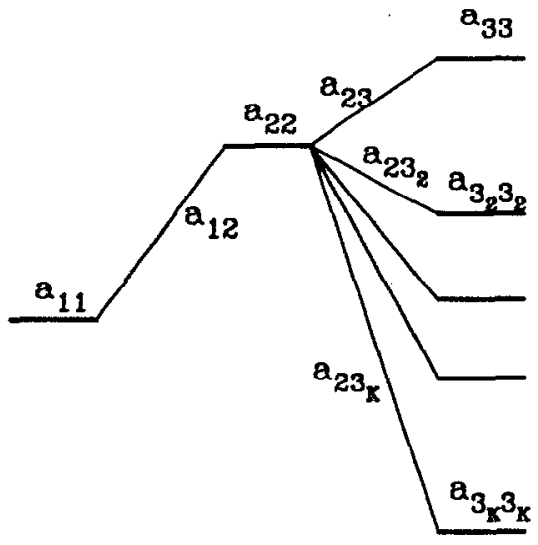

Tier 1 Tier 2 Tier 3

FIG. 5. A tree path.

$$
\begin{aligned}
R_{3}= & \alpha_{1}+\frac{a_{23}^{2}}{a_{22}-a_{11}} \\
& \times\left[1+\sum_{i=2}^{\kappa} \frac{a_{23_{i}}^{2}}{\left(a_{22}-a_{11}\right)\left(a_{33_{i}}-a_{11}\right)}\right],
\end{aligned}
$$

where $a_{23}$ is the coupling element between the tier-2 state and the the particular tier- 3 state whose value is being considered, $a_{23_{i}}, i=2,3, \ldots, K$ are the coupling elements between the tier-2 state and remaining $K-1$ tier-3 states, $a_{3,3}$ $i=2,3, \ldots, K$ are the positions of the latter $K-1$ tier-3 states. The tree path reduces to a simple path when $K=1$, and Eq. (13) reduces to Eq. (11) correspondingly.

Table II, given in Appendix B, shows the application of Eq. (3), and the second-order approximation given by Eq. (B18) in Appendix B, for several different tree paths. The comparison between the results obtained using Eq. (13) and those obtained by directly numerical calculation shows that Eq. (13) provides a good description for shift of resonance center for tree paths. In a way similar to that used for the simple paths, the width of resonance center for tree paths is found to be

$$
\tau_{\mathrm{trcc}}=\frac{4}{\sqrt{3}}\left|\frac{a_{12} a_{23}}{a_{22}-a_{11}-\delta}\right|,
$$

where $\delta$ is defined by Eq. (B12) (cf. Appendix B). If $\delta=0$ or if $\delta$ is negligible, $\mathrm{Eq}$. (14) reduces to the simple path case, as Eq. (12).

\section{EVALUATION FUNCTION}

In the present work, the evaluation function given by Eq. (3) incorporates, in addition to perturbation theory, the following factors.
TABLE II. Shift $R_{3}-\alpha_{1}$ of resonance center for trec path with 3 tier-3 state systems.*

\begin{tabular}{ccccc}
\hline \hline & & \multicolumn{3}{c}{ Shift $R_{3}-\alpha_{1}$} \\
\cline { 3 - 5 }$a_{23}$ & $a_{3_{2} 3_{2}}$ & Eq. (B18) & Eq. (B17) & Numerical \\
\hline \multirow{2}{*}{10.50} & 9200.00 & 0.55 & 0.55 & 0.56 \\
& 8820.00 & 0.61 & 0.61 & 0.64 \\
& 8810.00 & 0.66 & 0.68 & 0.73 \\
& 8805.00 & 0.77 & 0.86 & 0.79 \\
20.50 & 9200.00 & 2.12 & 2.12 & 2.21 \\
& 8820.00 & 2.31 & 2.34 & 2.37 \\
& 8810.00 & 2.53 & 2.61 & 2.76 \\
& 8805.00 & 2.95 & 3.28 & 3.22 \\
30.50 & & & & \\
& 9200.00 & 4.68 & 4.68 & 4.77 \\
& 8820.00 & 5.12 & 5.17 & 5.32 \\
& 8810.00 & 5.59 & 5.78 & 6.00 \\
& 8805.00 & 6.52 & 7.27 & 7.26 \\
& & & & \\
40.50 & 9200.00 & 8.26 & 8.26 & 8.36 \\
& 8820.00 & 9.04 & 9.12 & 9.32 \\
& 8810.00 & 9.86 & 10.19 & 10.40 \\
& 8805.00 & 11.50 & 12.82 & 11.90 \\
\hline \hline
\end{tabular}

- Tree path structure is shown in Fig. 2. Notations of matrix elements are defined in Eq. (B1). In this set of examples, $K=3, a_{11}=8800.0$, $a_{22}=9000.0, a_{12}=20.00, a_{33_{3}}=9100.00, a_{23_{2}}=20.0, a_{23_{3}}=10.00$ $\mathrm{cm}^{-1}, a_{23}$ and $a_{33}$ are parameters and given in the table, other elements are zero.

${ }^{\mathrm{b}}$ Quadratic term was used.

\section{A. Resonance effect of beam search and definition of $W_{2}$}

In general, in practical problems there may be more than one initial tier- 1 state and for each initial state there may be many tier- 2 states, or a tier- 2 state may even couple simultaneously with two or more initial states. In these cases the positions of the tier-1 rich states cannot be found simply according to Eq. (9), obtained using two-state perturbation theory. An alternative method, which we use in Part III, is to perform a prediagonalization after the beam search for the tier-2 states and to proceed as follows: The $N$ initial zerothorder states $\phi_{j}$ are assigned as the first $N$ states (tier-1 states) in the search procedure. A set of $(M-N)$ tier-2 states are then collected in the beam search process, all these $(M-N)$ zeroth-order states being denoted by $\phi_{j}, j=N+1, \ldots, M$. The $M$ eigenvectors obtained in the prediagonalization of these $M$ zeroth-order states are denoted by $\psi_{i}$,

$$
\psi_{i}=\sum_{j=1}^{M} c_{i j} \phi_{j}
$$

The population $\rho_{i}$ of tier-1 states in a $\psi_{i}$ is defined as

$$
\rho_{i}=\sum_{j=1}^{N}\left|c_{i j}\right|^{2} \text {. }
$$

When $\rho_{i}$ is greater than a given threshold (such as 0.1 ), which depends upon density of states and computer capacity, the corresponding eigenvalue of $\psi_{i}$ will be considered as a possible resonance center $\alpha_{1}$. 
The more strongly a tier-2 state $\phi_{j}$ $(j=N+1, N+2, \ldots, M)$ mixes with the initial states, the more important the tier-2 state will be. In Eq. (3) this importance is described by a weight factor $W_{2}$. For a practical AI search, there may be several initial states, i.e., tier-1 states. The number of tier- 2 states are even more, such as more than a hundred in the benzene system treated in Part III. It is sometimes difficult even to assign the parent state for a particular tier-2 state, since it may couple with several initial states. A weight factor for each of the $(M-N)$ tier-2 states, $\phi_{k}, k=N+1, \ldots, M$ denoted by $W_{2}^{(k)}$ is defined using a population analysis procedure,

$$
W_{2}^{(k)}=\sum_{i=1}^{M} \sum_{j=1}^{N}\left|c_{i j} c_{i k}\right|,
$$

where $i$ ranges over the $M$ prediagonalized (tier-1 plus tier2) states in Eq. (15) and $j$ over the $N$ initial zeroth-order tier1 states. Physically, this weight factor describes the relative amount of the $N$ initial states $\left\{\phi_{1}, \phi_{2}, \ldots, \phi_{N}\right\}$ mixed with a tier-2 state $\phi_{k}$ after the beam search on the tier-2 level at the starting point of a path proceeding from prediagonalized states $\psi_{i}$. The weight factor $W_{2}^{(k)}$ in Eq. (17) is written as $W_{2}$ in the evaluation function given by Eq. (3).

\section{B. The effect of later-accepted states on resonance centers}

As discussed previously, the influence of a tier- $n$ state on the global coupling picture depends on the proximity of that state to a resonance center. Therefore, the evaluation function needs to contain a factor, denoted by $\eta$, to characterize this effect. The value of $\eta$ is chosen to be given as in Eq. (5), where $R_{3}$ is given by Eq. (10). If the interaction between the tier-3 state and its parent state is very strong, i.e., if the element $a_{23}$ is very large, the corresponding resonance center is shifted from the value $\alpha_{1}$ of the prediagonalized tier-1 rich state (prediagonalized over tier- 1 and tier- 2 states). The position of this resonance center, for the values of $a_{33}$ after considering the effect from the latest accepted state, was calculated using Eq. (10), which in turn was derived in Appen$\operatorname{dix}$ A for a simple path.

\section{Choice of $\xi$ factor}

The magnitude of the evaluation function for a tier- $i$ state $\phi_{i}$ in a path depends, among other things, upon the mutual relationship between $a_{11}, a_{i-1, i-1}$ and $a_{i i}$. In the original equation, Eq. (1), and in Eq. (4a), the value of the evaluation function is controlled by the larger of the two quantities $\Delta E_{i 1}=\left|a_{i i}-a_{i 1}\right|$ and $\Delta E_{i, i-1}$ $=\left|a_{i i}-a_{i-1, i-1}\right|$. If the importance of $\phi_{i}$ were mainly determined by the smaller of the two quantities $\Delta E_{i 1}$ and $\Delta E_{i, i-1}$, the factor $\xi$ given in Eq. (4b) would be more appropriate.

Combining the above discussions, we obtain the AI search evaluation function shown in Eq. (3).

\section{Application to an actual system}

In this section we comment on the resonance centers $\alpha_{1}$ and $R_{3}$ that appear in Part III of this series, where the over- tone spectra and intramolecular dynamics of $\mathrm{CH}$ stretching of benzene are studied. In the application, tier-2 states were obtained using the beam search technique. The number of tier-2 states depends upon the system. For the overtone of $v_{\mathrm{CH}}=3$ of benzene, about 200 tier-2 states were found. A prediagonalization of the Hamiltonian using the initial states plus these tier-2 states was performed to obtain corresponding eigenvalues and eigenstates, which may be called tier-2 eigenstates. A population analysis technique was used to identify the tier-2 eigenstates which had major contributions from the initial states. The corresponding eigenvalues of these particular tier-2 eigenstates were regarded as the positions of resonance centers $\alpha_{1}$ on the tier-2 level for the system. The weight factors, denoted by $W_{2}$, of the tier-2 states which made an important contribution to the resonance centers were obtained, as were the $W_{2}$ 's of other states. The AI search procedure was continued so as to inspect higher tiers using the best-first search technique. When a new tier- 3 state is accepted, based on the value of the evaluation function, the influence of the tier- 3 state on the resonance centers was also examined. The position of a resonance center is shifted from $\alpha_{1}$ to $R_{3}$ as in Eqs. (11) and (13). Using Eq. (5) one may estimate the importance of a tier-3 state by comparing its energy with $R_{3}$, and change its "value" accordingly.

The application and comparison of the present evaluation functions to benzene are given in Part III of this series.

\section{ACKNOWLEDGMENTS}

This research was supported by the Caltech Consortium in Chemistry and Chemical Engineering; Founding Members: E. I. du Pont de Nemours and Company, Inc., Eastman Kodak Company, and Minnesota Mining and Manufacturing Company. It is a pleasure to acknowledge also the support of this research by a grant from the National Science Foundation.

\section{APPENDIX A: WIDTH OF TIER-3 RESONANCE RANGES}

The width of resonance range is described by the width at half-height of the resonance near the resonance center, $R_{3}$.

The partitioning method ${ }^{17}$ will be used to discuss this width for a simple path. Equation (7) is rewritten as

$$
\begin{aligned}
\mathbf{h}-e & =\left(\begin{array}{ccc}
a_{11}-E & 0 & a_{12} \\
0 & a_{33}-E & a_{23} \\
---- & ------- & --E
\end{array}\right) \\
& \equiv\left(\begin{array}{ll}
M_{a a} & M_{a b} \\
M_{b a} & M_{b b}
\end{array}\right),
\end{aligned}
$$

i.e., $M$ denotes the partitioned matrix. By defining a matrix $\bar{M}_{a a}$ by $^{17}$

$$
\bar{M}_{a a}=M_{a a}-M_{a b} M_{b b}^{-1} M_{b a},
$$

where the definition of $M$ matrices is given in Eq. (A1), we have

$$
\bar{M}_{a a}=\left(\begin{array}{cc}
\alpha_{1}-E & \beta \\
\beta & \alpha_{3}-E
\end{array}\right),
$$


where

$$
\begin{aligned}
& \alpha_{1}=a_{11}-\frac{a_{12}^{2}}{a_{22}-E}, \\
& \alpha_{3}=a_{33}-\frac{a_{23}^{2}}{a_{22}-E}, \\
& \beta=\frac{a_{12} a_{23}}{a_{22}-E} .
\end{aligned}
$$

The eigenvalues $E$ are obtained from det $\bar{M}_{a a}=0$. Exact resonance occurs when $\alpha_{3}=\alpha_{1}$. When the resulting value of $a_{33}$ is denoted by $R_{3}$, and the $E$ in the expression for $\alpha_{3}$ is replaced by $\alpha_{1}$, Eq. (10) is obtained from Eq. (A4).

The eigenvalue $E_{1}$ of the $\phi_{1}$-dominant eigenstate is easily found from Eq. (A3),

$$
E_{1}=\frac{1}{2}\left(\alpha_{1}+\alpha_{3}\right)+\frac{1}{2}\left(\alpha_{1}-\alpha_{3}\right) \sqrt{1+4 x^{2}},
$$

where

$$
x=\frac{\beta}{\alpha_{1}-\alpha_{3}} .
$$

The positive sign is chosen for $E_{1}$ in Eq. (A5), since we wish $E_{1}$ to tend to $\alpha_{1}$ when $\beta$ tends to zero; $E_{1}$ being the eigenvalue of the $\phi_{1}$-dominant state. Using the partitioning formulation the coefficient $c_{a}$ in the $\phi$ subspace matrix ${ }^{17}$

$$
\bar{M}_{a a} c_{a}=0 \text {, }
$$

with coefficients in $c_{a}$ being $c_{1 a}$ (the $\phi_{1}$-rich state) and $c_{3 a}$ (the $\phi_{3}$-rich state), we have

$$
c_{1 \alpha}^{2}=\frac{\beta^{2}}{\beta^{2}+\left(\alpha_{1}-E\right)^{2}} \text {. }
$$

At a resonance center, $\phi_{1}$ strongly mixes with $\phi_{3}$ such that $c_{1 a}^{2}=c_{3 a}^{2}=0.5$, as is seen in Figs. 3 and 4. Therefore, at "half height" $c_{1}^{2}$ is midway between 0.5 and unity (cf. Figs. 2 and 3 ), and hence is given by

$$
\mathbf{h}-\boldsymbol{c}=\left(\begin{array}{cc}
a_{11}-E & 0 \\
0 & a_{33}-E \\
---- & ---- \\
0 & 0 \\
\vdots & \vdots \\
0 & 0 \\
a_{12} & a_{23}
\end{array}\right.
$$$$
\left.\begin{array}{cccc}
0 & \cdots & 0 & a_{12} \\
0 & \cdots & 0 & a_{23} \\
\hdashline-- & - & -- & - \\
a_{3_{2} 3_{2}}-E & \cdots & 0 & a_{23_{2}} \\
\vdots & \ddots & \vdots & \vdots \\
0 & \cdots & a_{3_{k} 3_{k}}-E & a_{23_{k}} \\
a_{23_{2}} & \cdots & a_{23_{k}} & a_{22}-E
\end{array}\right) \equiv\left(\begin{array}{cc}
M_{a a} & M_{a b} \\
M_{b a} & M_{b b}
\end{array}\right)
$$

$$
c_{1 a}^{2}=\frac{3}{4}
$$

Combining Eqs. (A9), (A8), and (A5) we have the equation for $x$

$$
\left(1-\sqrt{1+4 x^{2}}\right)^{2}=\frac{4}{3} x^{2},
$$

where $x$ is defined by Eq. (A6). The roots of Eq. (A10) are

$$
x= \pm(\sqrt{3} / 2) \text {. }
$$

The values of $a_{33}$ corresponding to the roots of $x$ are

$$
a_{33}^{ \pm}=a_{11}-\frac{ \pm(2 / \sqrt{3}) a_{12} a_{23}+a_{12}^{2}-a_{23}^{2}}{a_{22}-E^{ \pm}},
$$

where $E \pm$ is the eigenvalue corresponding to $a_{33}^{ \pm}$. When, in a perturbation approximation the $E \pm$ in the denominator is replaced approximately by $a_{11}$, the width at half-height for the resonance is

$$
\tau=\left|a_{33}^{+}-a_{33}^{-}\right|=\frac{4}{\sqrt{3}}\left|\frac{a_{12} a_{23}}{a_{22}-a_{11}}\right|,
$$

which is the result given by Eq. (12).

\section{APPENDIX B: RESONANCE CENTER SHIFT FOR TREE PATHS}

We consider a tree path with $K$ tier-3 states as shown in Fig. 5. These tier-3 states are located at $a_{33}, a_{3_{2} 3_{2}}, \ldots, a_{3_{k} 3_{k}}$, respectively, where $a_{33}$ is the energy of the particular tier-3 state whose value is being considered. The coupling elements between the tier- 2 states and these tier- 3 states are $a_{23}$, $a_{23_{2}}, \ldots, a_{23_{k}}$, respectively. To study the shift of resonance centers we consider $a_{33}$ as being a variable, and other $a_{3,3}$ $(j=2, \ldots, k)$ being given parameters.

The partitioning method ${ }^{17}$ will be again used to study the shift of resonance center for tree paths. The Hamiltonian may be written in the partitioned form,

$$
\begin{aligned}
& M_{b b} \text { is a special form of the matrix } B \\
& B=\left(\begin{array}{cccc}
b_{11} & 0 & \cdots & b_{1 m} \\
0 & b_{22} & \cdots & b_{2 m} \\
\vdots & \vdots & \ddots & \vdots \\
b_{1 m}^{*} & b_{2 m}^{*} & \cdots & b_{m m}
\end{array}\right),
\end{aligned}
$$

where the only nonzero elements are $b_{i i}$ and $b_{i m}, i=1$ to $m$. It is not difficult to find its determinant $|B|$ and inverse $B^{-1}$, 


$$
\begin{aligned}
& B_{i j}^{-1}=\frac{1}{b_{j j}}+\frac{\left|b_{j m}\right|^{2}}{C b_{i j}^{2}}, \quad j=1,2, \ldots, m-1, \\
& B_{m m}^{-1}=\frac{1}{C}, \\
& B_{i j}^{-1}=\frac{b_{i m}^{*} b_{j m}}{C b_{i i} b_{i j}}, \quad i \neq j, i \neq m, j \neq m, \\
& B_{j m}^{-1}=-\frac{b_{j m}^{*}}{C b_{j j}}, \quad j=1,2, \ldots, m-1 .
\end{aligned}
$$

We again define a matrix $\bar{M}_{a a}$ by Eq. (A2), but where now $M_{a a}, M_{a b}, M_{b a}$, and $M_{b b}$ are given by Eq. (B1). $M_{a a}$ contains the quantity

$$
\begin{aligned}
M_{a b} M_{b b}^{-1} M_{b a} & =\left(\begin{array}{llll}
0 & \cdots & 0 & a_{12} \\
0 & \cdots & 0 & a_{23}
\end{array}\right) \cdot\left(M_{b b}^{-1}\right)\left(\begin{array}{cc}
0 & 0 \\
\vdots & \vdots \\
\vdots & \vdots \\
0 & 0 \\
a_{12} & a_{23}
\end{array}\right) \\
& =\left(M_{b b}^{-1}\right)_{22}\left(\begin{array}{cc}
a_{12}^{2} & a_{12} a_{23} \\
a_{12} a_{23} & a_{23}^{2}
\end{array}\right) .
\end{aligned}
$$

Applying Eqs. (B3)-(B9) to $\boldsymbol{M}_{b b}$ whose definition is given in Eq. (B1), we find

$$
\left(M_{b b}^{-1}\right)_{22}=\frac{1}{a_{22}-E-\delta},
$$

where

$$
\delta=\sum_{j=2}^{K} \frac{\left|a_{23,}\right|^{2}}{a_{3_{j} j}-E} .
$$

Substituting Eqs. (B10)-(B12) into Eq. (A2) we obtain

$$
\bar{M}_{a a}=\left(\begin{array}{cc}
\alpha_{1}-E & \beta \\
\beta & \alpha_{3}-E
\end{array}\right),
$$

where

$$
\begin{aligned}
& \alpha_{1}=a_{11}-\frac{a_{12}^{2}}{a_{22}-E-\delta}, \\
& \alpha_{3}=a_{33}-\frac{a_{23}^{2}}{a_{22}-E-\delta}, \\
& \beta=\frac{a_{12} a_{23}}{a_{22}-E-\delta} .
\end{aligned}
$$

At a resonance center, $a_{33}$, as a variable, satisfies $\alpha_{1}=\alpha_{3}$. Using this expression and Eqs. (B14) and (B15) we have the position of the resonance center being

$$
R_{3}=\left.a_{33}\right|_{\alpha_{1}=\alpha_{3}}=\alpha_{1}+\frac{a_{23}^{2}}{a_{22}-E-\delta}
$$

or

$$
R_{3}=\alpha_{1}+\frac{a_{23}^{2}}{a_{22}-E}\left[1+\frac{\delta}{a_{22}-E}+\frac{\delta^{2}}{\left(a_{22}-E\right)^{2}}+\cdots\right] .
$$

For a perturbation process $E \approx a_{11}$, and $\delta^{2}$ and higher terms in Eq. (B18) can be ignored, thus

$$
\begin{aligned}
R_{3}= & \alpha_{1}+\frac{\left|a_{23}\right|^{2}}{a_{22}-a_{11}} \\
& \times\left[1+\sum_{j=2}^{K} \frac{\left|a_{23_{j}}\right|^{2}}{\left(a_{22}-a_{11}\right)\left(a_{3,3}-a_{11}\right)}\right] .
\end{aligned}
$$

Table II shows the application of Eqs. (B19) and (B18) for several groups of different parameters. The results obtained from Eqs. (B19) and (B18) are in good agreement with the directly numerical data.

If tier-3 states are located far away from $\alpha_{1}$, i.e., $\left|a_{3,3},-a_{11}\right|>0$, the summation term in Eq. (B19) can be ignored. Thus, the tree path reduces to a single path, and Eq. (B19) reduces to Eq. (11), as is expected.

In a similar way shown in Appendix A, the width of resonance center for a tree path can be found being

$$
\tau_{\text {tree }}=\frac{4}{\sqrt{3}}\left|\frac{a_{12} a_{23}}{a_{22}-a_{11}-\delta}\right|,
$$

where $\delta$ is defined by Eq. (B12). If $\delta=0$ or delta is negligible, Eq. (B20) reduces to single path case, as Eq. (12).

${ }^{1}$ V. E. Bondybey, Annu. Rev. Phys. Chem. 35, 591 (1984); F. F. Crim, ibid. 35, 657 (1984); E. B. Stechel and E. J. Heller, ibid. 35, 563 (1984); I. Oref and B. S. Rabinovitch, Acc. Chem. Res. 12, 166 (1979); C. S. Parmenter, Faraday Discuss. Chem. Soc. 75, 7 (1983); M. S. Child and L. Halonen, Adv. Chem. Phys. 57, 1 (1984); J. D. McDonald, Annu. Rev. Phys. Chem. 30, 29 (1979); E. L. Sibert III, Int. Rev. Phys. Chem. 9, 1 (1990).

${ }^{2}$ P. J. Robinson and K. A. Holbrook, Unimolecular Reactions (WileyInterscience, New York, 1972); W. Forst, Theory of Unimolecular Reaction (Academic, New York, 1973).

${ }^{3}$ S. J. Klippenstein and R. A. Marcus, J. Chem. Phys. 93, 2418 (1990), and references cited therein; E. D. Potter, M. Gruebele, L. R. Khundkar, and A. H. Zewail, Chem. Phys. Lett. 164, 463 (1989), and references cited therein.

${ }^{4}$ W. F. Polik, D. R. Guyer, W. H. Miller, and C. B. Moore, J. Chem. Phys. 92, 3471 (1990).

'D. S. King, Adv. Chem. Phys. 50, 105 (1982); M. Quack, ibid. 395 (1982).

${ }^{6}$ L. J. Butler, E. J. Hintsa, and Y. T. Lee, J. Chem. Phys. 84, 4104 (1986); A. Mcllroy and D. Nesbitt, ibid. 91, 104 (1989).

${ }^{7}$ R. H. Page, Y. R. Shen, and Y. T. Lee, Phys. Rev. Lett. 59, 1293 (1987).

${ }^{8}$ S. M. Lederman, S. J. Klippenstein, and R. A. Marcus, Chem. Phys. Lett. 146, 7 (1988).

'S. M. Lederman and R. A. Marcus, J. Chem. Phys. 88, 6312 (1988).

${ }^{10}$ S. M. Lederman, V. Lopez, V. Fairen, G. A. Voth, and R. A. Marcus, Chem. Phys. 139, 171 (1989).

${ }^{11}$ J. V. Tietz and S. I. Chu, Chem. Phys. Lett. 101, 446 (1983); J. Chang and R. E. Wyatt, ibid. 121, 307 (1985); J. Chem. Phys. 85, 1826 (1986).

${ }^{12}$ A. Barr and E. A. Fcigenbaum, The Handbook of Artificial Intelligence, Vol. 1 (Heuris Tech, Stanford, 1981).

${ }^{13}$ J. Chang, N. Moiseyev, and R. E. Wyatt, J. Chem. Phys. 84, 4997 (1986).

${ }^{14}$ G. Jacucci and M. Rasetti, J. Phys. Chem. 91, 4970 (1987).

${ }^{15}$ B. G. Buchanan and E. A. Feigenbaum, in Reading in Artificial Intelligence, edited by B. L. Webber and N. J. Nilsson (Tioga, Palo Alto, 1981), pp. 313-322, and references cited therein.

${ }^{16}$ A. Nauts and R. E. Wyatt, Phys. Rev. Lett. 81, 2238 (1983); Phys. Rev. A 30, 872 (1984); C. Iung and C. Leforestier, Comput. Phys. Commun. 62, 135 (1991).

${ }^{17}$ P.-O. Lowdin, in Perturbation Theory and its Applications in Quantum Mechanics, edited by C. H. Wilcox (Wiley, New York, 1965), p. 255. 